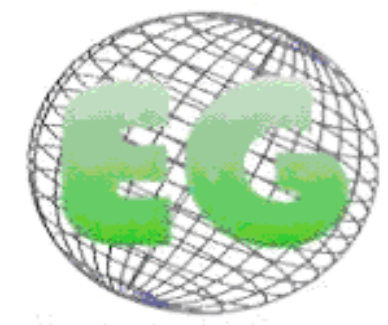

ISSN 1695-6141 $\mathrm{N}^{\circ} 26$

\title{
Efecto de una estrategia educativa participativa en el desarrollo del pensamiento crítico en estudiantes de enfermería
}

Effect of a participatory educational strategy on the development of critical thinking in nursing students

\section{${ }^{*}$ Carriles Ortiz, MG., ${ }^{* *}$ Oseguera Rodríguez, JF., "**Díaz Torres, Y., "***Gómez} Rocha, SA.

\begin{abstract}
*Lcda. en Enfermería. E-mail: martha.carriles@gmail.com ${ }^{* *}$ Doctor en Educación ***Maestra en Educación. ****Médico especialista en Medicina Familiar. Centro de Investigación Educativa y Formación Docente del Instituto Mexicano del Seguro Social, Delegación Querétaro. México.
\end{abstract}

Palabras clave: educación participativa; pensamiento crítico; proceso enfermero Keywords: participatory education; critical thinking; nursing process

\section{RESUMEN}

Introducción. La formación de la enfermera en México ha sido pasiva y tradicionalista, de ahí la preocupación de los docentes del área por reorientar la educación para dar respuesta al contexto que se vive actualmente, en donde se espera que la enfermera tenga un papel protagónico y participativo en la atención de los pacientes.

Objetivo. Identificar el grado de desarrollo del pensamiento crítico de la estudiante de enfermería en el cuidado de la paciente obstétrica posterior a una estrategia educativa participativa.

Material y métodos. Estudio de intervención, longitudinal en estudiantes de enfermería, en el que se realizó una estrategia educativa participativa con la finalidad de desarrollar el pensamiento crítico en el proceso enfermero y un grupo con enseñanza tradicional. El pensamiento crítico se evaluó a través de 4 indicadores en un instrumento elaborado ex profeso que fue validado por consenso de expertos metodológicos y disciplinares (K-R de 0.93). Análisis estadístico no paramétrico con Mac Nemar.

Resultados. Al comparar las calificaciones globales de ambos grupos, hubo un avance significativo en el grupo con intervención participativa $(p=0.031)$.

Conclusiones.- La estrategia educativa participativa favorece el desarrollo de habilidades cognitivas complejas como el pensamiento crítico 


\section{ABSTRACT}

Introduction: The training of the nurse has been passive and traditional hence the concern of teachers to refocus education in response to today's context where the nurse is expected to assume a leading and participative role in patient care.

Objective. To identify the degree of development of critical thinking in nursing students about the care of the obstetric patient following a participative educational strategy.

Material and methods. Longitudinal ilntervention study in nursing students, which included a participatory educational strategy to develop critical thinking in the nursing process in a traditionally taught group. Critical thinking was assessed using 4 indicators in a purpose built instrument that was validated by expert methodological and disciplinary consensus (KR of 0.93). Non parametric statistical analysis using Mac Nemar.

Results. When comparing the global ratings of both groups, there was a significant development in the participatory intervention group $(p=0.031)$.

Conclusions. The participatory educational strategy favors development of complex cognitive skills such as critical thinking.

\section{INTRODUCCIÓN}

Las enfermeras con práctica clínica se enfrentan día con día a una interminable variedad de situaciones con los pacientes y sus familias, cada situación representa una nueva experiencia, con problemas y cuidados individualizados, con distintos enfoques para la resolución de necesidades y diferentes perspectivas de la mejor forma de actuar. Ante estas situaciones clínicas es importante que la enfermera desarrolle un pensamiento crítico, para que el paciente reciba el mejor cuidado. El pensamiento crítico es un proceso complejo que se desarrolla a través de su participación activa frente a la educación ${ }^{(1)}$

La educación en enfermería, como proceso sociocultural, ha variado en el transcurso del tiempo y ha recibido influencias como la religiosa, tecnológica y científica, las dos primeras influencias abarcan gran parte de la historia de la formación de la enfermera mexicana, es fácil reconocer algunas características como la sumisión, la abnegación, la compasión y la disciplina extrema que la lleva a interiorizar actitudes pasivas limitándola en su iniciativa durante su desarrollo laboral, esta situación la convierte en ejecutora de indicaciones médicas, lo que la lleva a tecnificar exclusivamente su quehacer profesional, limitando el desarrollo de sus habilidades cognitivas como la reflexión, el análisis y la crítica. $\operatorname{lger}^{(2)}$ señala que en la práctica de enfermería, el profesional requiere del razonamiento crítico para tomar decisiones, ya que deberá reunir datos que definan con precisión necesidades reales y problemas potenciales, analizará las posibles intervenciones y elegirá la más apropiada para la mejoría del paciente.

La profesionalización constituye una estrategia y un proceso de cambio permanente para lograr las metas de la enfermería, acorde con las necesidades de atención a la salud en una sociedad que se encuentra en continua transformación. Dentro de los elementos fundamentales de la profesionalización se encuentra el desarrollo de pensamiento analítico y crítico para una mejor toma de decisiones y en base a estas un actuar profesional de la enfermera, este proceso exige cambios en el personal de enfermería en su forma de pensar y por ende en su forma de actuar, así como de apreciar el proceso salud - enfermedad, desde la perspectiva holística. ${ }^{(3)}$

El Instituto Mexicano del Seguro Social (IMSS) convencido de la necesidad de profesionalizar a su personal, por lo beneficios que de ello se derivan, establece un 
convenio con la Universidad Nacional Autónoma de México, con el objeto de implementar el Programa de Profesionalización en Enfermería, para personal de nivel técnico que labora en el IMSS a través del Sistema de Universidad Abierta (SUA) y conforme al esquema académico de la Escuela Nacional de Enfermería y Obstetricia, donde los participantes obtendrán el título de Licenciado en Enfermería y Obstetricia. ${ }^{(4)}$

El modelo educativo que se ha estructurado para la enseñanza de la enfermería en el contexto de la modalidad de educación abierta está conformado por un enfoque andragógico, la autodidaxia asistida, el estudio independiente y una postura constructivista del aprendizaje ${ }^{(5)}$; elementos que sustentan las formas de trabajo en los grupos de dicha modalidad, sin embargo, para el alumno es un desafío, pues aunque ya son adultos, con cierta experiencia laboral, madurez y compromiso, le es difícil desarrollar habilidades de estudio independiente, situación comprensible, ya que en nuestra sociedad mexicana las instituciones educativas desde el nivel preescolar hasta el superior, conservan un enfoque educativo tradicional con el cual se ha formado a la mayor parte del personal de la salud. Este modelo educativo genera la pasividad del alumno resaltando la autoridad y poder del profesor como poseedor del conocimiento, quien marca el ritmo del aprendizaje del alumno. Su relación con el alumno se circunscribe a sesiones discursivas o expositivas dentro de un aula, donde el alumno acepta la información como verdad absoluta con una actitud subordinante y en pocas ocasiones se cuestiona, o bien donde se asigna al alumno la exposición del tema, el cual en su actitud pasiva, realiza escasa búsqueda de información y en ocasiones se limita a expresar lo escrito en los textos sugeridos por el programa 0 profesor, y éste a "corregir" o aclarar las dudas ocasionalmente generadas en el alumno.

El programa de licenciatura en enfermería a través del SUA tiene como ejes transversales de su formación a la investigación y el proceso enfermero, este último es un método para ayudar a la resolución de la problemática de salud del individuo sano o enfermo, con el propósito de potenciar el ejercicio profesional, favorecer el desarrollo de las capacidades cognitivas, técnicas e interpersonales en enfermería. Sin embargo, en el contexto práctico, las enfermeras miran este proceso como una manera de hacer y no como una manera de pensar para actuar; es decir, en su doble componente intelectual y conductual $^{(6)}$, y es en el componente intelectual, en la forma de observar, analizar, reflexionar, donde la enfermera desarrolla habilidades cognitivas complejas como la identificación de datos significativos a las necesidades de los pacientes, la clasificación de los mismos, confrontación y emisión de juicios clínicos, que derivan en tomas de decisión para una propuesta de un plan de acción en beneficio de la salud del paciente, favoreciendo así el desarrollo del pensamiento crítico que nos lleva por ende a una mejor actitud y desempeño profesional.

El pensamiento crítico es el proceso activo, cognitivo y deliberado, empleado para examinar el propio pensamiento e implica la utilización de la mente para reflexionar, efectuar deducciones, conclusiones y tomar decisiones. La Asociación Americana de Filosofía identifico habilidades básicas del pensamiento crítico que cuando se aplican a la enfermería son útiles para mostrar la naturaleza compleja de la toma de decisiones clínicas, se requiere además el desarrollo de hábitos personales como el formularse preguntas, permanecer bien informado, ser honesto al enfrentarse a prejuicios personales y estar siempre dispuesto a considerar las opiniones de los demás, la capacidad para desarrollar y aplicar estas habilidades requieren de la experiencia y la consideración cuidadosa del conocimiento desarrollado en el cuidado clínico de los pacientes. ${ }^{(1)}$

Sobre el pensamiento crítico en el área de enfermería algunas autoras han investigado como es el caso de Caballero ${ }^{(7)}$ quien realizó un estudio para identificar y evaluar las destrezas del pensamiento crítico en alumnos de primero y segundo año de la carrera de enfermería, 
estudio realizado en Santiago de Chile, donde concluye algunos aspectos de gran pertinencia en relaciona las estrategias educativas, donde se debe fomentar la búsqueda de información desde todos los puntos de vista, analizar críticamente situaciones determinadas y las fuentes de información, buscar el descubrimiento, la comprensión y confrontación del punto de vista de otros y desarrollar una postura inquisitiva.

De igual forma Gordon ${ }^{(8)}$ realiza un estudio en donde a través de una estrategia de enseñanza dirigido a un grupo de estudiantes de enfermería en un curso introductorio y enfocado al desarrollo de destrezas de pensamiento crítico, los resultados de este estudio proveen datos empíricos sobre la efectividad de enseñar las destrezas de pensamiento crítico y sugiere la inclusión de éstas en el contenido curricular de los programas de enfermería como curso específico para el desarrollo de las mismas.

Sobre el desarrollo de habilidades cognitivas complejas se han realizados algunos trabajos de investigación en el Instituto Mexicano del Seguro Social, en el área docente, en su mayoría con la finalidad de evaluar el grado de aptitud clínica del personal o estudiantes de enfermería, entendiendo a la aptitud clínica como "el conjunto de habilidades complejas involucradas en la detección y resolución de problemas clínicos suscitados en la práctica cotidiana de la enfermera". De los estudios revisados se pueden señalar el publicado por García A. (9) "Evaluación de la aptitud clínica a través de la metodología del proceso de enfermería" y el de García G. ${ }^{(10)}$ "Aptitud clínica en estudiantes de enfermería ante situaciones clínicas complejas", con resultados semejantes, en donde el personal de enfermería y estudiantes son clasificadas en un nivel muy bajo de aptitud clínica, lo que sugiere una práctica profesional basada en rutinas más que en acciones reflexivas y críticas.

Estudios más recientes como el que realiza Rivera (11) "Aptitud clínica en estudiantes de enfermería en el proceso de atención materno-infantil" o bien el de Torres-Carrasco ${ }^{(12)}$ "Aptitud clínica en la atención de enfermería prenatal y perinatal del binomio madre-hijo de bajo riesgo" en los que realizan una intervención educativa con enfoques participativo y tradicional, los resultados vislumbran beneficios, con avances poco significativos, en el desarrollo de la aptitud clínica de los participantes que fueron intervenidos con un enfoque participativo y cómo el enfoque tradicional en algunos de los casos lleva a un retroceso al estudiante, e infieren que la aptitud clínica reflexiva no está lo suficientemente estimulada en el ámbito educativo del país.

De acuerdo a la revisión bibliográfica realizada, el enfoque participativo ofrece una nueva alternativa de establecer el proceso educativo; los docentes en enfermería buscan actualmente el desarrollo de algunas habilidades en el alumno como rescatar su experiencia para la reflexión, el cuestionamiento de su quehacer profesional, el enjuiciamiento y discriminación de la información para la toma de decisiones, y la autocrítica, elementos que favorecen la elaboración del conocimiento, un pensamiento crítico y en consecuencia una mejor proyección profesional y humana. Ante esta conceptualización es necesario redefinir la orientación en la educación de la enfermera, buscando e incluyendo nuevas alternativas que rompan el paradigma tradicional , por lo que el propósito de este estudio es evaluar el grado de avance en el pensamiento crítico del proceso enfermero en estudiantes de séptimo semestre del curso de nivelación de licenciatura en enfermería y obstetricia SUA, en la atención a mujeres con embarazo, parto y puerperio de riesgo, posterior a dos estrategias educativas, una con enfoque participativo (EEP) y otra con un enfoque tradicional (EET). 


\section{MATERIAL Y MÉTODOS}

Se trata de un estudio de intervención, longitudinal, con estudiantes de licenciatura del SUA, realizado en el Hospital General Regional No. 1 del IMSS, Querétaro y en el Hospital General de Zona no. 3 en San Juan del Río, se incluyeron dos grupos naturales del séptimo semestre de la licenciatura en enfermería y obstetricia, los grupos denominados grupo de intervención (Gl) y grupo control (GC) integrados por 7 y 6 estudiantes respectivamente y que cursaban la asignatura de obstetricia II.

Las estudiantes debieron contestar en forma completa y correcta el instrumento de evaluación pre y post intervención educativa, contar con una asistencia del $90 \%$ de las sesiones educativas y estar vigentes en el programa académico para no ser eliminadas del estudio.

El pensamiento crítico se evaluó en el proceso enfermero en la atención de la paciente obstétrica de riesgo, entendiendo al pensamiento crítico del proceso enfermero como la capacidad cognitiva para identificar, reconocer y analizar los datos, signos y síntomas significativos que denoten respuestas individualizadas a problemas de salud reales 0 potenciales que derivan en la construcción de un diagnóstico enfermero o problema interdependiente, y que son la base para la planeación de acciones pertinentes y oportunas para el mantenimiento y restablecimiento del bienestar del individuo.

Se establecieron cuatro indicadores, el primero integración diagnóstica que se refiere al juicio clínico de enfermería que se realiza en base al análisis e interpretación de la información obtenida en la valoración del paciente, identificando las necesidades reales y/o potenciales y su asociación con los factores etiológicos o de riesgo; el segundo indicador se refiere a la identificación de complicaciones reales y/o potenciales toda información sobre el estado de salud de la paciente, que se obtienen de fuentes directas e indirectas que identifican a problemas interdependientes $(\mathrm{PI})$ y/o complicaciones potenciales(CP); el tercer indicador, intervención pertinente de enfermería, hace alusión a las acciones de enfermería más convenientes, y oportunas para la satisfacción de las necesidades reales y/o de riesgo identificadas en el paciente de forma individual y considerando las capacidades y limitaciones que conllevan su estado mórbido; el cuarto indicador, colaboración en la terapéutica médica, incluye las acciones a través de las cuales la enfermera trabaja en coordinación con el equipo médico para el tratamiento de $\mathrm{PI}$ y $\mathrm{CP}$ en el proceso de embarazo, parto y/o puerperio.

El diseño del instrumento se conformó por cuatro casos clínicos reales problematizados de los padecimientos prevalentes que afectan a la mujer embarazada en el contexto social que se desempeñan las participantes. De los 176 ítems iniciales se aplicaron 156 posterior a la ronda de expertos con un concenso de tres expertos metodológicos y cuatro de los cinco disciplinares. El tipo de respuesta fue verdadero, falso y no sé, donde la calificación global está dada por la suma de respuestas correctas y resta de la suma de las respuestas incorrectas, y las respuestas No sé son nulas. Se construyó una escala de cinco niveles para el pensamiento crítico, muy bajo, bajo, medio, alto y muy alto, dividiendo la escala en rangos excluyentes y a partir de 25 aciertos ya que se consideró los efectos explicables por el azar a través de la prueba de Pérez-Padilla y Viniegra, para instrumentos de falso, verdadero y no sé, ${ }^{(13)}$

Para estimar la consistencia del instrumento se aplicó una prueba piloto en una población de diez alumnas con características similares a las participantes. Se obtuvo un coeficiente de 0.93 con la fórmula 20 de Kuder Richardson. 
Las actividades para la estrategia educativa participativa (GI) fueron intra y extra aula; en una primera aproximación, las estudiantes individualmente debían leer y reflexionar sobre uno de los casos clínicos real estructurado y problematizado por el docente con una guía anexa para su resolución, con la intención de que la estudiante indagara nueva información a través de la revisión bibliográfica, la contrastara con su experiencia y elaborara un argumento para sus respuestas. En el aula cada alumna confrontó sus opiniones con las demás a través de una mesa de discusión, donde el docente fue el moderador con orientación en las ideas clave a fin de propiciar la discusión, la reflexión y autocrítica entre las estudiantes.

En el grupo de la estrategia educativa tradicional (GC), predominaron las actividades tradicionales en nuestro contexto educativo formativo, exposición del docente o asignación del tema al alumno, con resolución de dudas por el docente. Las tareas extra-aula fueron resúmenes de lecturas asignadas o temas revisados en clase los cuales eran entregadas al profesor.

El instrumento se aplicó en una medición antes y después de las intervenciones educativas en ambos grupos y fue aplicado por los docentes responsables de cada grupo. El análisis estadístico fue con Wilcoxon para medianas y la de $U$ de Mann Whitney, Mac Nemar, con un valor de $p<0.05$ como significativo.

\section{RESULTADOS}

Al realizar la comparación de las calificaciones globales, ambos grupos tuvieron un comportamiento similar antes y después de la estrategia educativa con cierta ventaja para el GI como se observa en el Cuadro I.

Cuadro I. Calificaciones globales obtenidos por las estudiantes antes y después de la estrategia educativa

\begin{tabular}{|c|c|c|c|}
\hline Grupo & $\begin{array}{c}\text { Antes } \\
\text { Med } \\
\text { (Min-Max) } \\
\end{array}$ & $\begin{array}{c}\text { Después } \\
\text { Med } \\
\text { (Min-Max) } \\
\end{array}$ & ${ }^{*} p$ \\
\hline $\begin{array}{c}\text { GI } \\
(n=7)\end{array}$ & $\begin{array}{c}59 \\
(26-68)\end{array}$ & $\begin{array}{c}88 \\
(69-106)\end{array}$ & 0.018 \\
\hline $\begin{array}{c}\mathrm{GC} \\
(n=6)\end{array}$ & $\begin{array}{c}60 \\
(52-75)\end{array}$ & $\begin{array}{c}67.5 \\
(56-81)\end{array}$ & 0.027 \\
\hline${ }^{* *} \mathbf{p}$ & 0.567 & 0.010 & \\
\hline
\end{tabular}

Calificación teónica máxima 156

${ }^{*}$ p Prueba de rangos asignados de Wilcoxon

**p Prueba U de Mann Whitney

Al comparar las medianas de los dos grupos en cada uno de los indicadores establecidos (Cuadro II), antes de las estrategias educativas no se encontró diferencia estadística, sin embargo al comparar las medianas posteriores a la intervención se observa como en el GI la integración diagnóstica y colaboración con la terapéutica médica hubo significancia estadística ( $p<0.05 U$ de Mann Whitney). En los puntajes de las calificaciones pre y post 
intervención en el avance al interior del grupo, en tres de los indicadores el Gl tuvo un avance significativo estadísticamente, en la integración diagnóstica ( $p=0.018$ con Wilcoxon), identificación de complicaciones reales o potenciales $(p=0.034)$ y colaboración con la terapéutica médica $(p=0.018)$.

Cuadro II. Calificaciones por indicador obtenidos por las estudiantes antes y después de la estrategia educativa

\begin{tabular}{|c|c|c|c|c|c|c|c|c|c|c|c|c|}
\hline \multirow[b]{2}{*}{ Grupo } & \multicolumn{3}{|c|}{ Integración diagnóstica } & \multicolumn{3}{|c|}{$\begin{array}{c}\text { Identificación de } \\
\text { complicaciones reales } 0 \\
\text { potenciales }\end{array}$} & \multicolumn{3}{|c|}{$\begin{array}{l}\text { Intervención pertinente de } \\
\text { enfermería }\end{array}$} & \multicolumn{3}{|c|}{$\begin{array}{l}\text { Colaboración con la } \\
\text { terapéutica médica }\end{array}$} \\
\hline & $\begin{array}{l}\text { Antes } \\
\text { Med } \\
\text { (Min- } \\
\text { Max) }\end{array}$ & $\begin{array}{c}\text { Después } \\
\text { Med } \\
\text { (Min- } \\
\text { Max) }\end{array}$ & ${ }^{*} \mathrm{p}$ & $\begin{array}{l}\text { Antes } \\
\text { Med } \\
\text { (Min- } \\
\text { Max) }\end{array}$ & $\begin{array}{l}\text { Después } \\
\text { Med } \\
\text { (Min- } \\
\text { Max) }\end{array}$ & ${ }^{*} \mathrm{p}$ & $\begin{array}{l}\text { Antes } \\
\text { Med } \\
\text { (Min- } \\
\text { Max) }\end{array}$ & $\begin{array}{c}\text { Después } \\
\text { Med } \\
\text { (Min- } \\
\text { Max) }\end{array}$ & ${ }^{*} \mathrm{p}$ & $\begin{array}{l}\text { Antes } \\
\text { Med } \\
\text { (Min- } \\
\text { Max) }\end{array}$ & $\begin{array}{l}\text { Después } \\
\text { Med } \\
\text { (Min- } \\
\text { Max) }\end{array}$ & ${ }^{*} \mathrm{p}$ \\
\hline $\begin{array}{c}\text { GI } \\
(\mathrm{n}=7)\end{array}$ & $\begin{array}{c}8 \\
(1-11)\end{array}$ & $\begin{array}{c}17 \\
(10-26)\end{array}$ & $\begin{array}{c}0.01 \\
8\end{array}$ & $\begin{array}{c}20 \\
(7-26)\end{array}$ & $\begin{array}{c}26 \\
(19-31)\end{array}$ & 0.034 & $\begin{array}{c}15 \\
(7-25)\end{array}$ & $\begin{array}{c}17 \\
(10-23)\end{array}$ & 0.588 & $\begin{array}{c}13 \\
(6-17)\end{array}$ & $\begin{array}{c}27 \\
(22-29)\end{array}$ & 0.018 \\
\hline $\begin{array}{c}\text { GC } \\
(n=6)\end{array}$ & $\begin{array}{c}9 \\
(4-14)\end{array}$ & $\begin{array}{c}8 \\
(6-16)\end{array}$ & $\begin{array}{c}0.58 \\
1\end{array}$ & $\begin{array}{c}22.5 \\
(21-24)\end{array}$ & $\begin{array}{c}25 \\
(21-27)\end{array}$ & 0.068 & $\begin{array}{c}21 \\
(17-23)\end{array}$ & $\begin{array}{c}17 \\
(17-22)\end{array}$ & 0.041 & $\begin{array}{c}10 \\
(6-14)\end{array}$ & $\begin{array}{c}16 \\
(10-18)\end{array}$ & 0.028 \\
\hline${ }^{* *} \mathrm{P}$ & 0.662 & 0.018 & & 0.221 & 0.942 & & 0.220 & 0.712 & & 0.219 & 0.003 & \\
\hline
\end{tabular}

*p Prueba de rangos asignados de Wilcoxon

**p Prueba U de Mann Whitney

Con relación a la distribución de las estudiantes de acuerdo a los niveles establecidos de pensamiento crítico en el proceso del cuidado enfermero, se puede observar en el cuadro III, hubo un avance en el $57 \%$ de las estudiantes del Gl contra un avance del $17 \%$ de las alumnas en el GC.

Cuadro III. Nivel del pensamiento critico en el proceso enfermero obtenido por las estudiantes de enfermería SUA en el cuidado de la paciente obstétrica, antes y después de la estrategia educativa

\begin{tabular}{|c|c|c|c|c|}
\hline \multirow[b]{2}{*}{ Categoría } & \multicolumn{2}{|c|}{ Antes } & \multicolumn{2}{|c|}{ Después } \\
\hline & $\begin{array}{c}\text { GI }(\mathbf{n}=7) \\
\%\end{array}$ & $\begin{array}{c}\mathrm{GC}(\mathrm{n}=6) \\
\%\end{array}$ & $\begin{array}{c}\text { GI }(n=7) \\
\%\end{array}$ & $\begin{array}{c}\text { GC }(n=6) \\
\% \\
\end{array}$ \\
\hline $\begin{array}{c}\text { Muy alto } \\
(132-156)\end{array}$ & - & - & & \\
\hline $\begin{array}{c}\text { Alto } \\
(106-131)\end{array}$ & - & - & $\begin{array}{c}1 \\
(14 \%)\end{array}$ & \\
\hline $\begin{array}{c}\text { Medio } \\
(79-105)\end{array}$ & - & - & $\begin{array}{c}3 \\
(43 \%)\end{array}$ & $\begin{array}{c}1 \\
(17 \%)\end{array}$ \\
\hline $\begin{array}{c}\text { Bajo } \\
(52-78)\end{array}$ & $\begin{array}{c}4 \\
(57 \%)\end{array}$ & $\begin{array}{c}6 \\
(100 \%)\end{array}$ & $\begin{array}{c}3 \\
(43 \%)\end{array}$ & $\begin{array}{c}5 \\
(83 \%)\end{array}$ \\
\hline $\begin{array}{c}\text { Muy bajo } \\
(25-51)\end{array}$ & $\begin{array}{c}3 \\
(43 \%)\end{array}$ & - & - & \\
\hline $\begin{array}{l}\text { Explicable por azar } \\
\qquad(\varangle 25)\end{array}$ & - & - & - & \\
\hline Total & $7(100 \%)$ & $6(100 \%)$ & $7(100 \%)$ & $6(100 \%)$ \\
\hline
\end{tabular}


En el cuadro IV se observa el criterio de avance del 25\% esperado posterior a la intervención educativa, con significancia estadística para el GI (Mac Nemar $p<0.05$ )

\begin{tabular}{|c|c|c|c|c|}
\hline Grupo & Sin cambios & Ganancia** & Descenso & ${ }^{*} \mathrm{p}$ \\
\hline GI $n=7$ & 1 & 6 & 0 & 0.031 \\
\hline $\mathrm{GC} n=6$ & 5 & 1 & 0 & 1.000 \\
\hline
\end{tabular}

\section{DISCUSIÓN}

En estudios previos realizados en el Instituto Mexicano del Seguro Social, por personal que se encuentra directamente involucrado en la formación de las enfermeras, han explorado la utilidad de evaluar habilidades del pensamiento a través de instrumentos conformados con casos clínicos reales en los que la intención es rescatar la experiencia del sujeto y la reflexión sobre esta misma. ${ }^{(9,10,11,12)}$

Actualmente una de las preocupaciones de los profesores es el contar con instrumentos que permitan medir algunos aspectos que poco se consideran, pero que se reconoce la necesidad de evaluar, como lo es el desarrollo del pensamiento crítico en el ámbito de enfermería, diseñar instrumentos que evalúen más allá de la capacidad memorística de los estudiantes. Evaluar las habilidades complejas del pensamiento implica evaluar el ejercicio de actividades cognitivas como el análisis y la reflexión de la experiencia propia para la toma de decisiones en el quehacer profesional; para el diseño de instrumentos con estos alcances se requiere de un proceso largo y complejo, desde la selección y seguimiento de los casos clínicos para la estructuración del instrumento, hasta la búsqueda de expertos metodológicos y disciplinares para la validación del mismo.

Otro aspecto importante a discutir es la forma en cómo se realiza la estrategia educativa bajo este enfoque, ya que ésta dinámica al interior del grupo, la forma de trabajo y tareas asignadas a los estudiantes, las relaciones humanas que establece el asesor con los estudiantes donde se rompan los esquemas tradicionales de profesor-expositor-poseedor del conocimiento y alumno-receptor de información, así como un ambiente de respeto por la opinión de los participantes, determinan en gran medida el avance de los alumnos en la elaboración de su propio conocimiento, por ello la importancia de que el asesor posea la habilidad para conducir a los estudiantes a retomar y reflexionar sobre su experiencia clínica y que realicen una autocrítica, facilitar en el alumno la necesidad de buscar, analizar, discutir y criticar información para re-elaborar su conocimiento y elegir la decisiones más acertadas y propuesta de intervención más pertinente en su actuar profesional. 
Para la enfermera docente es una tarea difícil, se requiere de una formación que fortalezca la postura docente, sus habilidades didácticas y actitud crítica; para ser creador de ambientes en donde, como señala Viniegra ${ }^{(14,15)}$, las condiciones sociales, institucionales y grupales sean propicias para la reflexión a través de la discusión, el debate y la confrontación de puntos de vista; donde el dogmatismo y sectarismo cedan el paso y surjan nuevas propuestas y argumentos que rivalicen en relevancia fuerza y pertinencia.

En este estudio se demuestra la ventaja de la intervención educativa con enfoque participativo en el desarrollo del pensamiento crítico a través del proceso enfermero en las estudiantes, tal como lo demostró Arteaga ${ }^{(16)}$ que señala cómo la pedagogía contemporánea considera que la mejor forma de activar el proceso cognitivo para el desarrollo del pensamiento crítico de los estudiantes es la utilización de la enseñanza problémica, en donde el alumno al enfrentarse a un hecho problematizado busca la mejor decisión para

resolverlo. Sin embargo como puntualiza Hawes ${ }^{(17)}$ lo primero es asumir que el pensamiento crítico existe y es una práctica habitual en el quehacer humano, con diferentes grados de complejidad según el dominio teórico en que se inserta y diversos grados de competencia de acuerdo a quienes lo ejercen.

El sustento teórico en el cual se fundamentó este estudio: el enfoque participativo de la educación y "la crítica de la experiencia" , nos da argumentos para plantear la necesidad de reorientar la forma de mirar la educación y la forma de ejercer la docencia en nuestros ámbitos institucionales educativos y de salud, con el firme propósito de participar como docentes y favorecer la elaboración del conocimiento de nuestros estudiantes, contribuyendo así a la formación de profesionales de la enfermería, analíticos, críticos y participativos en la resolución de la problemática del cuidado.

\section{CONCLUSIÓN}

El enfoque participativo ofrece una nueva alternativa de establecer el proceso educativo en el área de enfermería, y favorecer el desarrollo de habilidades cognitivas complejas en las estudiantes, como el pensamiento crítico en el proceso enfermero y donde se vea esta metodología básica del quehacer profesional, el proceso enfermero, no como una forma de hacer, sino como una forma de pensar para actuar, incluyendo el componente intelectual y no solo el conductual.

Es necesario que la enfermera-docente cree espacios en donde la reflexión de la experiencia de los estudiantes para la elaboración del conocimiento permita mejorar las capacidades de su actuar cotidiano, donde la teoría y la práctica se encuentren cabalmente vinculadas y se favorezca el desarrollo del pensamiento crítico, de esta forma se vea reflejado en el desempeño de los estudiante en el momento de la práctica, al realizar acciones fundamentadas en el análisis, la crítica y autocrítica.

Finalmente considerando que la efectividad de una intervención educativa radica en la medida que sus efectos permanecen a lo largo del tiempo, se sugiere realizar evaluaciones a mediano y largo plazo al grupo de estudiantes intervenidas.

\section{BIBLIOGRAFÍA}

1. Potter, P. Perry A. Fundamentos de enfermería, 5a Ed. Elsiver-Mosby, España, 2001, pp283-296. 
2. Iger, P. Proceso y diagnóstico de enfermería, Mc Graw-Hill Interamericana, 3ª̣. Ed. España. 1997, pp. 22-23.

3. Calderón M. La profesionalización en enfermería.

En: http://www.hospitalgeneral.salud.gob.mx/descargas/pdf/enfermeria/arti 09.pdf $10 / 02 / 09$

4. Matus-Miranda R, Poblano- Rosas O, Avances y perspectivas del Programa de Licenciatura en Enfermería y Obstetricia en el Instituto Mexicano del Seguro Social, Rev Enferm IMSS 2005; 13(2): 105-110

5. Espinosa, A.; Rodríguez, S. y Pacheco, A. Evolución del Sistema Universidad Abierta y a Distancia de la ENEO y su impacto social, UNAM, ENEO, México, 2000.

6. Zavala- Rodríguez MR. Perspectiva cualitativa de la práctica docente, construcción y uso del conocimiento por alumnos de licenciatura en enfermería. Rev Enferm IMSS 2005; 13(2):83-89

7. Caballero E, Arratia Figueroa A. Evaluación e identificación de destrezas del pensamiento crítico en alumnos de primero y segundo año de la carrera de EnfermeríaObstetra. Santiago de Chile: Escuela de Enfermería, Pontificia Universidad Católica; 2001.

8. Gordon M. Congruency in defining critical thinking by nurse educators and non-nurse scholars. J Nur Educ. 2000;39(8):340-51.

9. García, A., González, R. "Evaluación de la aptitud clínica a través de la metodología del proceso de enfermería", Rev Enferm IMSS 2001 9(3): 127-131.

10. García, G., Matus R. "Aptitud clínica en estudiantes de enfermería ante situaciones clínicas complejas”, Rev Enferm IMSS 2002 10(3): 131-136.

11. Rivera, S. Viniegra L. Matus R. Aptitud clínica en estudiantes de enfermería en el proceso de atención materno-infantil. Rev Enferm IMSS 2004; 12(3): 137-146.

12. Torres-Carrasco L. Viniegra L. Matus R. Aptitud clínica en la atención de enfermería prenatal y perinatal del binomio madre-hijo de bajo riesgo, .Rev Enferm IMSS 2005; 13(2):63-69

13. Pérez Padilla R., Viniegra L., "Método para calcular la distribución para las calificaciones esperadas por azar en un examen de tipo falso, verdadero o No sé", Rev Invest Clin 1989 41: 375-379.

14. Viniegra L. La crítica y la práctica médica. Rev. Inst. Nal. Enf. Resp. Mex. 1995; 8(4): 263-266.

15. Viniegra L. La Crítica, aptitud olvidada por la educación. 1aㅡ Ed, IMSS, México. 2000

16. Arteaga J. Proceso docente-educativo en el ciclo clínico como sistema integral (conferencia). Cuba. 2002.

17. Hawes G. Pensamiento crítico en la formación universitaria. Proyecto Mecesup TAL 01/01. Colombia, 2003. En: www.sep.ucr.ac.cr/GESTION/PENSAM1.PDF 19/ 02/ 09 